\title{
REVUE DES REVUES
}

\author{
Mónica Raísa Schpun (ed.), « 1908-2008. Le centenaire de l’immigration \\ japonaise au Brésil. L'heure des bilans ", dossier, Cahiers du Brésil contempo- \\ rain (Paris, Maison des Sciences de l'homme), 71/72, 2008 [parution 2009], 318 p., \\ résumés trilingues (fr., port., angl., p. 299-311), ISSN : 0989-5191.
}

Ce numéro double des Cahiers du Brésil contemporain de 2008 est extrêmement utile, puisqu'il aborde, en langue française, l'histoire de l'immigration japonaise dans le pays. Celle-ci commença très exactement en 1908 par l'arrivée d'un premier contingent d'environ 750 travailleurs japonais appelés par les fazendas de café. Aujourd'hui, leurs descendants, les Nikkeis, au nombre d'environ 1,7 million d'âmes, sont des Brésiliens dont l'identité n'est plus principalement définie par leur origine, mais celle-ci reste néanmoins présente, ne serait-ce que dans le regard des autres Brésiliens. Par ailleurs, un phénomène inverse a commencé dans les années 1980, à savoir le «retour » de Nikkeis dans un Japon qu'ils n'avaient jamais connu, dans l'optique primordialiste des autorités nippones: les Dekasseguis devaient avoir les mœurs des Japonais, puisqu'ils en avaient le «sang», et donc n'avoir aucune difficulté d'intégration dans le pays. Les entreprises japonaises cherchaient ainsi à freiner l'immigration asiatique destinée aux métiers les moins rémunérés sur l'archipel, en recrutant d'autres «Japonais ». En 2005, ils étaient 215 500, y constituant la troisième communauté étrangère après les Coréens et les Chinois, et devant les Philippins.

Une large partie des articles réunis dans cette livraison ont déjà été publiés ailleurs, en portugais (Brésil), mais comme le souligne Mónica Raísa Schpun dans l'introduction, telle est justement l'une des fonctions des Cahiers : «rendre accessible en français des pans significatifs de la réflexion brésilienne contemporaine qui y trouvent sinon la seule, en tout cas la principale porte d'entrée en France » (p. 9). Que les revues Nouveaux Mondes/Novos Mundos/Nuevos Mundos, Caravelle et Lusotopie ne se sentent pas vexées, les Cahiers du Brésil contemporain sont effectivement fort utiles! Cette livraison l'est en particulier, parce que le centenaire de cette immigration n'a pas été l'occasion d'un regain d'études en sciences sociales sur le sujet, sauf rares exceptions (l'une est mentionnée en fin de volume en recension ${ }^{1}$ ).

Le volume s'ouvre par la publication, en forme d'hommage, d'un article de 1963, de Ruth Corrêa Leite Cardoso, pionnière des études sur les Nikkeis du Brésil, «L'agriculteur et le professionnel libéral chez les Japonais du Brésil»

\footnotetext{
${ }^{1}$ Il s'agit de Jeffrey Lesser, Uma diaspora descontente: os nipos-brasileiros e os significados da militancia étnica 1960-1980, São Paulo, Paz e terra, 2008, 293 p. (éd. originale anglaise : A Discontent Diaspora: Fapanese-Brazilians and the Meanings of Ethnic Militancy, 1960-1980, Duke University Press, 2007); par ailleurs, la thèse de Ruth Corrêa Leite CARdoso, soutenue en 1972 à l'Université de São Paulo, n’a été publiée qu'en 1998 : Estrutura familiar e mobilidade social: estudo dos japoneses no estado de São Paulo, édité par Masato Ninomiya, São Paulo, Kaleidos-Primus, 1998 ; les deux recensions sont de Helena Prado (p. 285-298).
} 
(p. 11-20), ensuite discuté par Célia Sakurai dans son «Commentaire à "L'agriculteur ..." de Ruth Corrêa Leite Cardoso » (p. 21-24). Il s'agit surtout de montrer comment les travaux de Ruth C.L. Cardoso ont ouvert les chemins d'une meilleure compréhension des processus d'intégration des immigrants dans la complexité de leur relation à la société d'accueil, n'ignorant pas « une bonne dose de sensibilité pour capter les innombrables variables qui se rapportent au thème étudié » (p. 24).

L’article de l’organisatrice du volume, Mónica Raísa Schpun, «L’immigration japonaise au Brésil. Six générations en un siècle» (p. 25-36) brosse, comme il se doit en début de l'ouvrage, l'histoire de la communauté depuis les débats sur l'opportunité de l'immigration asiatique au Brésil commencés dès que l'abrogation de l'esclavage fut envisagée (comment et par qui remplacer la main-d'œuvre servile?), aux débuts difficiles de Japonais qui parfois n'avaient jamais été des paysans avant de travailler dans les fazendas de café, à leur urbanisation difficile et jusqu'à la situation actuelle où le niveau de vie moyen de la communauté semble nettement plus élevé que la moyenne brésilienne (p. 51). Cependant, ce qui domine, contrairement au regard porté sur eux, c'est l'hétérogénéité, tant sociale, économique que culturelle, de ces descendants japonais.

Le second article, de Rogério Dezem, porte sur les "Nuances du "Jaune". Éléments formateurs de l'imaginaire sur le Japonais au Brésil» (p. 57-86). On le voit, il ne s'agit pas ici des immigrés japonais eux-mêmes, mais du regard que portent sur eux les Brésiliens blancs. L'article aborde l'origine de ce regard, qui était principalement porté sur le "Jaune », c'est-à-dire au départ sur les Chinois, au stéréotype fort négatif. Les Japonais s'en distinguèrent ensuite quelque peu. L'auteur relate un épisode fort intéressant, celui du «scrutin russo-japonais » organisé par O Malho auprès de ses lecteurs en avril 1904 et qui eut un succès considérable (plus de dix mille lettres de réponse). Or, à la question, confuse il est vrai («Pour lequel des deux pays manifestez-vous vos votes?») de savoir qui allait gagner la guerre, ou auquel des deux pays allait la sympathie des lecteurs, plus des quatre cinquièmes désignèrent le Japon. La sympathie pour le pays neuf, refusant l'hégémonie occidentale, était évidente. Mais c'était avant l'arrivée des travailleurs immigrés japonais ... Les stéréotypes refirent ensuite surface, jusqu'à l'ostracisme officiel dont les Japonais du Brésil furent victimes à partir de 1942 quand G. Vargas déclara la guerre aux puissances de l'Axe. Les Chinois restèrent cependant toujours stéréotypés à un rang inférieur à celui des Japonais, quoique le «péril jaune » fût désormais attribué à ces derniers.

L'une des raisons de la méfiance envers les Japonais, surtout lors de l'Estado Novo de Vargas, fut qu'ils développaient avec acharnement des écoles pour transmettre leur culture propre, mettant ainsi en question la construction de la nation. C'est cette histoire que brosse Hiromi Shibata dans l'article «Les écoles des immigrants japonais. Organisations de l'engagement et orientation japonisante » (p. 85-122). L'essor des écoles en langue japonaise fut stoppé net par la réglementation de l'Estado Novo, surtout à partir de 1937: interdiction des subventions étrangères, obligation de n'enseigner qu'en portugais à tout enfant de moins de 14 ans. Néanmoins, la transmission de la langue des ancêtres a continué de manière semi-clandestine mais efficace. Le nationalisme brésilien a alors renforcé la tendance $\mathrm{au}$ nationalisme japonais face au danger de «destruction de la race japonaise» 
orchestrée par le varguisme. L'entrée en guerre du Brésil en 1942 a bien sûr accentué cette tendance. Après la guerre, des écoles en nombre moindre et des associations ont repris une activité, mais qui ne visait désormais plus à former des Japonais, mais à transmettre la culture japonaise à des Brésiliens d'ascendance japonaise.

L'article de Rosangela Kimura aborde un épisode particulier de cette histoire, «Shindô Renmei. Défaite de 1945 et conflits intracommunautaires chez les Japonais du Brésil » (p. 123-150). Il s'agit de l'histoire politique de l'éclatement de la communauté japonaise pendant la guerre au Brésil, et surtout à la fin de la guerre, quand une partie d'entre elle crut que la nouvelle de la défaite du Japon était une gigantesque opération de désinformation et attaqua comme traîtres ceux qui reconnaissaient la défaite, allant jusqu'à la pratique d'assassinats. L'origine du Shindô Renmei vient de la politisation de l'effort de préservation de l'identité culturelle, radicalisée encore par les spoliations et l'ostracisme de la période 1942-1945. Outre son aspect politique, il y eut aussi un aspect social, les "défaitistes » étant en général plutôt membres de la bourgeoisie nippo-brésilienne, et les « victoiristes » étant de condition plus humble et ne croyant pas que, avec un Empereur divin, l'annonce de la défaite ait pu parvenir au Brésil sous la forme d'un simple communiqué du ministère japonais des Affaires étrangères transmis à la Croix Rouge internationale de Genève, puis transitant par la Croix Rouge argentine avant d'être transmis à quelques notables de la communauté. Néanmoins, après la guerre, de ce mouvement transformé surgit aussi le mouvement pour les droits civils des Japonais du Brésil, contre les discriminations dont ils souffraient.

Célia Sakuray aborde la situation de l'après-guerre dans "L'immigration des Japonais au Brésil dans l'après-guerre (1950-1980)» (p. 151-174). Cette émigration est naturellement due à la situation difficile du pays après la défaite. Côté brésilien, c'était un moment où le ministère du Travail mettait l'accent sur la nécessité d'une immigration spécialisée dans certaines compétences. Le Brésil rétablit des relations diplomatiques avec le Japon en 1952 et l'immigration japonaise fut « officialisée » par un décret du président Goulart en 1963. La période resta celle de stéréotypes à l'encontre des Nippo-brésiliens, mais du moins la «discrimination raciale » est considérée comme «anachronique, au moins quand elle est officielle » (p. 1592). Par ailleurs cela heurtait «la seule démocratie raciale existant dans le monde entier » (idem). L'industrialisation, l'urbanisation, l'entrée d'entreprises étrangères au Brésil modifièrent ensuite l'univers social des Japonais vers des métiers citadins. La « communauté » devient encore plus hétérogène, car les «nouveaux Japonais » arrivés, déjà qualifiés, ne ressemblent pas aux deux ou trois premières générations arrivées au Brésil, mais pour autant était fort différente des descendants brésiliens de celles-là. Mais, pour la société brésilienne, ils restaient tous « les Japonais ».

Fábio Ricardo Ribeira évoque ensuite une situation parfaitement contemporaine, dans «L'exotisme domestique. Sexualité et masculinité chez les Nippo-descendants » (p. 175-199). Remarquons tout d'abord que «nippo-descendant» est un concept qui n'avait pas été utilisé par les auteurs précédents. On pourrait du reste le discuter, tout comme fut fortement discuté en France celui de «luso-descendant». Non point qu'il n'y ait des gens qui « descendent» de telle ou telle origine, mais

\footnotetext{
${ }^{2}$ Citation d'un numéro de la Revista de Imigração e Colonização, Rio de Janeiro, 1952.
} 
celle-ci est-elle déterminante pour la définition de leur vie sociale? L'auteur paraît le penser dans son étude de cas. En pratique, il n'étudie pas la sexualité et la masculinité des «Nippo-descendants », mais les pratiques sociales et amoureuses des seuls homosexuels de cette origine. L'étonnement de l'auteur provint du fait que, fréquentant le milieu des gays, lesbiennes et sympathisants dans les bars, les boîtes de nuit et autres lieux de socialisation, il n'y rencontrait jamais de « Nippodescendants ». N'y avait-il donc pas de Nippo-descendants homosexuels? Il y en avait bien sûr, mais qui n'apparaissaient pas dans les lieux ouverts précités, mais fréquentaient assidûment les réseaux internet et, en général, ne tissaient de relations qu'avec des partenaires habitant d'autres villes, rarement à proximité. L'auteur conclut en constatant que «ces individus, en construisant une homosexualité imprégnée de morale japonaise, et où la figure du pénis et l'acte sexuel ne se trouvent pas au centre des attentions, nous permettent de penser de nouvelles façons de vivre la (l'homo)sexualité et, ce faisant, de remettre en cause d'autres images, tout aussi stéréotypées » (p. 198).

Une autre situation contemporaine, mais non relative à la même classe d'âge, est relatée par Guita Grin Debert, dans son article «Comment dissoudre l'ethnicité dans la consanguinité. Les vieux d'une maison de retraite de la communauté japonaise à São Paulo» (p. 201-228). Cette maison est la plus ancienne créée au Brésil pour la communauté japonaise, dirigée par une association catholique et semble à tous égards modèle, puisque les personnes âgées qui y sont pensionnaires peuvent y être comprises dans leur langue (alors que leurs descendants ne la parlent plus), maintenir des liens avec leur parentèle souvent éloignée et, surtout, y travaillent tous aux tâches d'entretien ( [Quand je vais chez mes] enfants, je ne fais que manger, je grossis et je n'ai rien à faire ! », entrevue, p. 221). Ainsi les liens ethniques (la nipponité) y ressemblent à des liens familiaux.

Les deux derniers articles du dossier abordent les Dekasseguis, à savoir les Nippo-brésiliens « revenus » au Japon et leur accueil par leur « société d'origine ». Lili Kawamura aborde «La discrimination sociale et culturelle dans la migration de Brésiliens au Japon» (p. 229-255). Le premier obstacle auquel ces immigrants ont dû faire face fut le mythe de l'homogénéité sociale et culturelle des "Japonais ", du fait qu'ils ont été appelés au Japon, en fonction de cette croyance, pour éviter l'essor de l'immigration asiatique. Or leur comportement au travail, face aux normes de production, la relation au patron, la dissociation de la vie familiale et de celle au travail qu'ils pratiquaient, tout cela montra très vite qu'ils étaient des Brésiliens. Mais ces problèmes ne sont en général pas encore affrontés de manière collective, malgré un début de développement d'écoles, d'associations et d'ONG.

Yumi Garcia dos Santos aborde "Les familles étrangères au Japon. Une étude comparative sous le prisme du genre » (p. 257-281). Elle évoque en effet successivement les communautés chinoise, coréenne, philippine et brésilienne avant de s'attarder sur cette dernière, en examinant en particulier le ratio hommes-femmes et le profil social des uns et des autres. Ainsi, l'immigration philippine est-elle à $82 \%$ féminine, ultra-précaire (contrats de six mois) et spécialisée dans les métiers d'entertainment (boîtes de nuit, etc., mais, contrairement à la rumeur, la prostitution est très minoritaire). La communauté coréenne - première communauté étrangère du pays - est naturellement d'origine coloniale et, tout comme la chinoise, est formée d'une majorité de femmes, mais en proportion moindre que dans le cas 
précédent : respectivement 54 et $60 \%$ en 2005. Les femmes chinoises deviennent souvent les épouses de paysans japonais restés célibataires. À l'inverse, dans la communauté brésilienne (formée de descendants de Japonais, mais aussi de leurs conjoint(e)s d'autres origines et enfants), le ratio est de $55 \%$ pour les hommes et de $45 \%$ pour les femmes. Les mariages sur place avec des Japonais ne sont pas rares $(21 \%$ chez les deux sexes) et ne souffrent pas d'une proportion de divorces pire que celle des couples «purement» brésiliens au Japon (environ $30 \%$ ). Les Brésiliennes du Japon accouchent bien plus tôt que les Coréennes et Japonaises, semblant reproduire ce comportement au Brésil - ce qui n'est pas pour étonner pour des primo-arrivants. La scolarisation est un énorme problème, certains enfants n'étant pas du tout scolarisés malgré l'obligation scolaire au Japon : mais les parents ne comprennent pas la langue et craignent des comportements intolérants à l'égard de leurs enfants à l'école, y compris de la part des professeurs. De ce fait, des écoles brésiliennes voient le jour. L'auteure termine son article en se définissant elle-même comme une «japonaise atypique» pour laquelle cet article a été un moyen d'attirer l'attention sur « une caractéristique peu connue du Japon: celle d'une société multiethnique et internationale en lente formation au début du $\mathrm{xx}^{\mathrm{e}}$ siècle et nettement accélérée depuis ces vingt dernières années [remettant en cause] le mythe du Japon uniracial» (p. 277). Le Japon, un nouveau Brésil ? Il faudra encore attendre un peu...

On terminera en exprimant quelques regrets, et un reproche. L'organisatrice, Mônica Raísa Schpun, prévient dès la première page de son introduction que ce volume des Cahiers ne prétend combler le manque d'études en sciences sociales sur les Japonais du Brésil, même s'il veut contribuer à le relativiser. En langue française au moins, la contribution est notable. Cependant, les auteurs sont tous des historiens, des sociologues et des anthropologues : on aurait pu espérer une pluridisciplinarité plus large, notamment vers la géographie et les sciences politiques. Sur la base des données de l'Institut brésilien de géographie et statistiques et de ses recensements, ne pouvait-on présenter une analyse statistique, spatiale, voire cartographiée, de la communauté japonaise? Les enjeux politiques (sauf à propos de l'épisode de la Seconde Guerre mondiale) sont totalement absents : n'aurait-il pas été intéressant de savoir si les Nippo-brésiliens votent comme les autres? Sur le plan des relations internationales, l'existence des Nikkeis et des Dekasseguis a-t-elle une influence sur les relations bilatérales nippo-brésiliennes? Enfin, la mixité des couples formés par des Nikkeis, qui se développe, concernet-elle aussi les Brésilien(ne)s noir(e)s ou s'agit-il seulement d'intermariages avec des Brésilien(ne)s blanc(he)s? On attendra donc un deuxième volume pour obtenir des réponses à ces questions!

Le reproche concerne la mauvaise qualité de quelques traductions (bourrées de lusitanismes), déplorable dans le cas de Rosangela Kimura ${ }^{3}$. Parfois, il s'agit même

\footnotetext{
${ }^{3}$ «. . . le gouvernement a décidé d'exécuter la fiscalisation d'organisations . . .» (p. 126); «. . il était pendant l'État Nouveau qui a eu lieu la "période de disqualifications, plus radical [sic] des différences..."» (p. 127); «... la tâche de la Police politique [...] qui avait comme besogne registrer tous les types de comportement...» (p. 127); «Le climat de suspicion [...], comme il s'est passé sur la côte de Paraná....» (p. 127); «C'était le prêtre Guido Del Toro [...] le responsable de faire passer le message ...» (p. 129-130); «... treize ans d'actuation» (p. 132);
} 
de la syntaxe, voire de la plus élémentaire ponctuation : cela concerne en particulier l'article, absolument massacré, de Lili Kawamura ${ }^{4}$. Dans une revue qui paraît depuis vingt-deux ans, ce problème de traitement des articles est difficilement compréhensible et écorne la qualité remarquable de l'ensemble ici présenté.

Fuillet 2009, Michel Gahen

«. . 30 mille membres registrés ...» (p. 132); «. . la série a commencé avec la prison, septembre 1944, du principal chef ...» (p. 134); «Le Component de l'Armée impériale japonaise ...» (p. 134); «... des salaires assez compensateurs ...» (p. 143); «Étant donné qu'il est le moment de plusieurs récoltes de céréales, la prison de centaines de chefs de famille peut les faire tout perdre...» (p. 144). Bref, parfois, il faut savoir le portugais pour comprendre ce qui est écrit en ... franguês.

${ }^{4}$ On citera un seul passage, mais tout l'article est ainsi : «Si, lors de la première moitié du $\mathrm{xx}^{\mathrm{e}}$ siècle, le groupe nippo-brésilien était constitué par des personnes aux caractéristiques ethniques et culturelles similaires, influencés par les modèles culturels traditionnels japonais. C'est lors de la seconde moitié de notre siècle que nous pouvons observer des transformations dans ce contingent de population qui l'aurait mené à une différenciation culturelle et même ethnique. Cela à cause des multiples formes d'insertion de ses membres dans la société brésilienne. Sous cette perspective, à partir des années soixante, on peut affirmer que la population nikkei s'était déjà très éloignée du concept de communauté homogène. Parce que différente et distante de l'ensemble de la société brésilienne. Contrariant ainsi la vision exprimée par le sens commun au Brésil. Bien qu'elle comprenne des descendants de Japonais, et ayant donc un héritage culturel nippon, la parcelle nikkei de la population brésilienne en regroupe plusieurs types. À partir de ceux du type physique asiatique du Japon, avec les coutumes, les comportements et les valeurs de l'ère Meiji. Celles-ci ont été apportées par les immigrés japonais, vivant encore dans les campagnes et les petites villes. Jusqu'aux dénommés sansei, yonsei et métisses (avec leur physionomie occidentalisée).» (p. 233-234). 